\title{
MICRO-STRUCTURING OF SILICON BY PULSED-LASER ABLATION UNDER REACTIVE ATMOSPHERES.
}

\author{
S. JESSE*, A. J. PEDRAZA*, J. D. FOWLKES*, J. D. BUDAI** AND D. H. LOWNDES** \\ *Dept. of Materials Science and Engineering, The University of Tennessee, Knoxville, TN \\ 37996-2200 ajp@utk.edu. \\ **Solid State Division, Oak Ridge National Laboratory, Oak Ridge, TN 37831-6056.
}

\begin{abstract}
Micro-holes hundreds of $\mu \mathrm{m}$-deep below the initial surface surrounded by $40-\mu \mathrm{m}$-tall microcones protruding over the initial surface form in silicon as a response to multiple UV pulsed-laser irradiation in an $\mathrm{SF}_{6}$ atmosphere. The micro-holes and micro-cones are arranged in a characteristic ensemble displaying a self-organized pattern. Similarly, deep-holes and micro-column ensembles form when the irradiation is performed in an oxygen-rich atmosphere. The formation mechanism of microcolumns and microcones in these reactive atmospheres has been studied using scanning electron microscopy, profilometry and x-ray diffraction. The growth of cones under $\mathrm{SF}_{6}$ atmospheres was studied in-situ using an ICCD gated camera coupled to a long distance microscope. The images obtained with the ICCD camera show that the fluorescent plume is correlated with the amount of ablated material. There is also a strong correlation between the plume and the growth of the cones. The cones grow in the region where the laser-generated plume is intense. When the flux of silicon-rich material ceases because the holes are very deep the balance between redeposition and ablation is tilted toward the latter and the cones begin to recede. Laser ablation is not only a function of the nominal fluence but also of the surface roughness and microstructure. These results strongly support the growth mechanism whereby laser-ablated silicon-rich molecules and clusters are transported to the tip and side of the cones by the lasergenerated plasma.
\end{abstract}

\section{INTRODUCTION}

Laser-induced ablation can be due to several processes including mechanical spallation, expulsion of small droplets from a transiently laser-melted layer, desorption of atoms and ions, and evaporation [1]. The presence of a reactive atmosphere can augment ablation by producing compounds that are easily removed by laser irradiation. The action of the laser on the target can produce very drastic changes in surface topography. When silicon is irradiated in air with 1000 laser pulses at a power of $130 \mathrm{MW} / \mathrm{cm}^{2}$, a dense array of high aspect ratio columns forms on the surface. Theses columns are $\sim 20 \mu \mathrm{m}$ long and $2-3 \mu \mathrm{m}$ in diameter (figure 1) [2-7].

In silicon, we have observed that evolution of cone-like structures is extremely dependent on the irradiation atmosphere. An array of very large and complex conical structures around deep microholes is formed if the irradiation is performed in sulfur hexafluoride $\left(\mathrm{SF}_{6}\right)[6,8,9]$. Both, the columnar and conical features just described, protrude above the original wafer surface. A possible mechanism that explains these morphological details is redeposition of surrounding ablated silicon on top of the emerging features $[2,6,9]$. In this paper we present in-situ studies of the evolution of the laser-induced plume and the growth of cones together with the deepening of microholes. A relation between the fluorescent plume and the growth and dissolution of cones, and the deepening of microholes is established.

\section{X-RAY ANALYSIS}

The cones formed during laser irradiation of (111) silicon substrates in $\mathrm{SF}_{6}$ are single crystal and have the same orientation as the substrate. However columns formed during irradiation in 
oxygen-rich atmospheres are polycrystalline in nature. Most probably the nature of the depositing species determines whether single crystal cones or polycrystalline columns are formed. The top and sides of cones and the top of columns are melted during laser irradiation. Complete resolidification of the melted regions takes place a few hundred nanoseconds following the laser pulse [10]. It has been well established that the solidification of silicon after nanosecond laser melting takes place by the motion of a solid/liquid interface moving from the non-melted substrate [11]. This process of melting and resolidification would generate a single crystal with the same orientation as the substrate. The growth of cones seems to follow this pattern. However, for irradiation in oxygen-rich atmospheres small oxide particles generated during laser irradiation seem to be deposited on the liquid silicon together with silicon at the column's tip. Significantly high concentration of oxygen is detected both at the tip and walls of the columns [2]. These small oxide particles may nucleate silicon and favor the growth of small crystals during the solidification process.

\section{LASER-INDUCED MICROSTRUCTURAL EVOLUTION OF SILICON IN SF 6 ATMOSPHERES}

The evolution of a cone structure on a silicon substrate during irradiation in $\mathrm{SF}_{6}$ was followed using a long focal distance microscope attached to an ICCD camera. The irradiation was performed at a laser fluence of $3.1 \mathrm{~J} / \mathrm{cm}^{2}$, measured on a flat surface, and at an $\mathrm{SF}_{6}$ pressure of $500 \mathrm{mbar}$. In a first series of images one picture was taken after each pulse always having the same delay time of $55 \mathrm{~ns}$. The delay time is measured from the time the laser pulse initially reaches the substrate to the initial opening of the ICCD gate. Acquisition of images while maintaining a constant delay time is referred to as continuous mode. This mode is used primarily to characterize changes associated with the number of laser shots. Another method used to obtain images is by sequentially increasing the delay time from one exposure to the next. This is referred to as sequential mode and is used to characterize events during a single laser pulse. The nominal time that the ICCD gate is open is the pulse width. The part of the laser plume that is visible is due only to the plume components that are in excited electronic states. Non-emitting atoms, ions, molecules, clusters and micro-droplets are not observed in these images. [12].

Images taken in a continuous mode show no detectable plume until the sample has been irradiated with $\sim 500$ laser pulses. The profile of a silicon specimen laser exposed to 400 pulses at a fluence of $3 \mathrm{~J} / \mathrm{cm}^{2}$ shows a very shallow crater and a relatively smooth surface and an ablation rate of only $1.2 \mathrm{~ns} /$ pulse [13]. This ablation rate is very small and is consistent with the ICCD images. After 600 pulses a rough wavelike surface develops and the ablation plume is visualized in the ICCD images (Fig.1a). As the roughness increases a characteristic structure of microcones and microholes develops. Although the cone structure eventually covers the laser illuminated region in a fairly uniform manner, cone evolution is not necessarily homogeneous at any given time.

Figure $1 \mathrm{a}, 1 \mathrm{~b}$ and $1 \mathrm{c}$ shows the images at 600,700 , and 800 pulses taken in a continuous mode after a delay time of $55 \mathrm{~ns}$ and a $4.5 \mathrm{~ns}$ pulse width. All the images in fig. 1 are almost edge-on, that is, the normal to the irradiated surface is at an angle of $\sim 95^{\circ}$ with the normal to the plane of observation. In this way the line of cones at the edge of the substrate is clearly observed without major interference from the cones that are being formed beyond the wafer edge. The edge of the sample is fully irradiated as it is located at the center of the laser spot. The tip of the cones in Figs. 1a,b and c can be seen as small bright spots elevated over the initial surface of the wafer. In Figs. 1d, 1e and 1f, after 900, 1300 and 1700, the cone profiles are well defined. The fluorescent plume reaches up to 40 to $60 \mu \mathrm{m}$ over the tips of the cones and it is higher in the area where the cones are growing. We have observed that once the cones reach a certain height the plume tends to move towards another area of the laser exposed region and the cones quickly start to develop in the newly plume immersed region. Together with the development of the distinctive cones deep holes are formed. Bright spots are observed below the target surface when holes perforate the substrate's lateral surface. These bright spots at the bottom of the holes indicate an increase in the plume fluorescence (Figs. 1f and 1g). The ablation rate in the holes as measured using SEM [8] and the 
ICCD images is $1.4 \mu \mathrm{m}$ per pulse. This is much larger than the ablation rate measured on a flat surface by profilometry, thus giving clear indication of the correlation between the observed plume and the amount of ablated material. A similar ablation rate in the microholes has been measured with an SEM in specimens irradiated at $2.4 \mathrm{~J} / \mathrm{cm}^{2}$ and at a background pressure of 500 mbar of $\mathrm{SF}_{6}$ [8]. Figure 1f shows that the cones protrude up to $30 \mu \mathrm{m}$ above the initial surface indicating that silicon-rich molecules and clusters were transported from the ablated microholes onto the cones.

The cones grow by the influx of silicon-rich material that emanates from the microholes onto the molten tips, but at the same time the laser beam is ablating the cone tips. These experiments and others $[6,8]$ clearly indicate that the amount of ablated material that is deposited on the tip is larger than the amount of silicon that is being ablated from the tip. However, as the microholes deepen the fluorescent plume continuously recedes and after 1700 pulses it reaches the level of the cones tips (Fig. 1f). As the microholes deepen even further the fluorescent plume disappears. The same cones that protrude over $30 \mu \mathrm{m}$ after the first 1700 laser shots are now, after 22000 shots, fully ablated and the microstructure roughness is now below the initial surface (Fig. 1g).

We also studied the plume evolution as a function of pressure by recording in sequential mode images between 800 and 900 laser pulses. Each image was captured $1 \mathrm{~ns}$ later than the preceding one. These sequential images were then studied, and the average distance from the top of the cones to the most distant edge of the plume was measured. The plume velocity, measured at the beginning of the ablation process, is a function of the ambient pressure. As the pressure increases the initial plume velocity first decreases, then increases reaching a maximum of $1.1 \mu \mathrm{m} / \mathrm{ns}$ at $500 \mathrm{mbar}$, and finally decreases again for higher pressures (Fig. 2). This behavior probably indicates that two competing phenomena are influencing the plume velocity.

\section{DISCUSSION AND CONCLUSIONS}

Laser ablation is not only a function of the fluence but also of the surface roughness and microstructure. Very smooth surfaces produce ablation rates under $\mathrm{SF}_{6}$ that are over three orders of magnitude less than when the surface contains microholes and microcones. However as the microholes become very deep the ablation rate decreases again. A computer simulation which takes into account multiple beam reflections on the cone and microhole walls shows that the multiple reflections converging towards the bottom of the microhole strongly increase the intensity at the bottom of the hole. For this calculation the shape of the cone and the microhole were taken from a cross sectional SEM image. Assuming a reflectivity of 0.6 for silicon illuminated with $248 \mathrm{~nm}$ wavelength laser light, it is obtained that the bottom of the hole receives a $400 \%$ increase in intensity. The intensity at the hole bottom would thus be $12 \mathrm{~J} / \mathrm{cm}^{2}$ and would strongly increase the ablation of silicon. Moreover the $\mathrm{SF}_{6}$ inside the hole is also subjected to multiple irradiations promoting further decomposition and increasing the amount of fluorine formed. As discussed in earlier work, fluorine forms volatile compounds with silicon further enhancing the ablation process $[6,8]$. The number of reflections in the walls of the holes increases and the intensity decreases, as the holes deepen. But even when the walls reach a depth of $\sim 300 \mu \mathrm{m}$ the contribution of the beam reflections to the fluence received at the bottom of the hole is very significant thus promoting ablation. The very bright spots seem to indicate that a very strong ablation takes place at the bottom of the holes (Fig 1g). It has to be pointed out that this calculation assumes smooth internal walls. We have observed that cluster and microdroplets are laid at the walls of the microholes and microcones increasing the wall roughness. The roughness of the walls will tend to scatter light in all directions decreasing the amount of radiation reaching the bottom of the microholes.

The fluorescent plume is not visible when the ablation rate is very small. As the microholes appear and the ablation rate increases a laser-generated plume extending 40 to $60 \mu \mathrm{m}$ over the

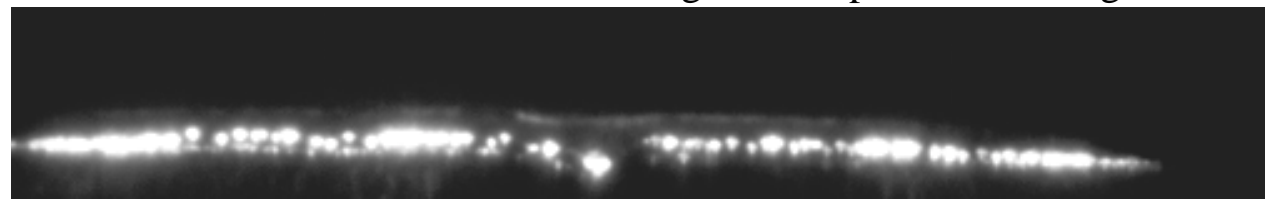




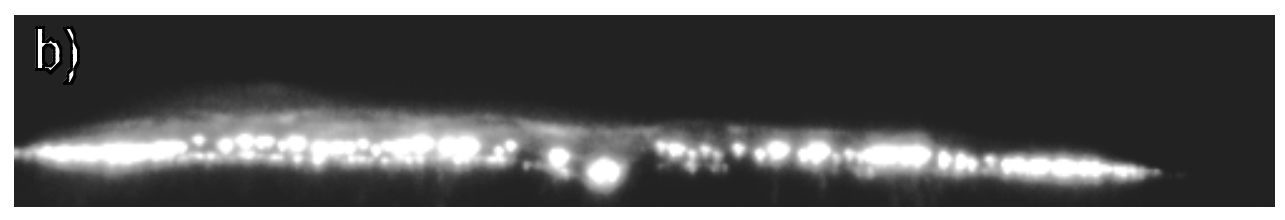

700 shots

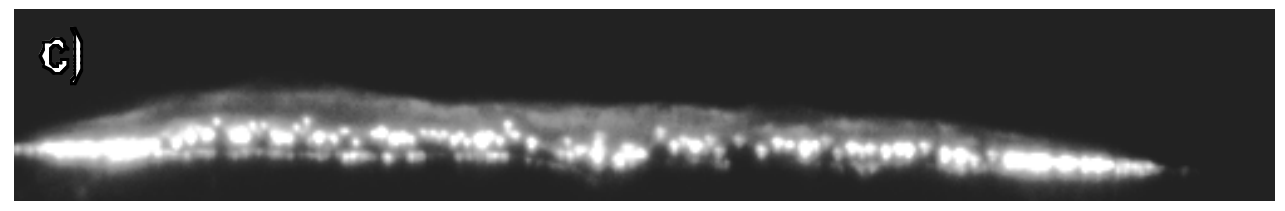

800 shots

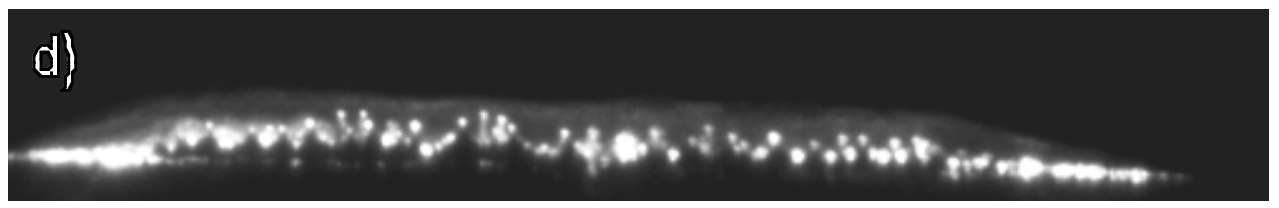

900 shots

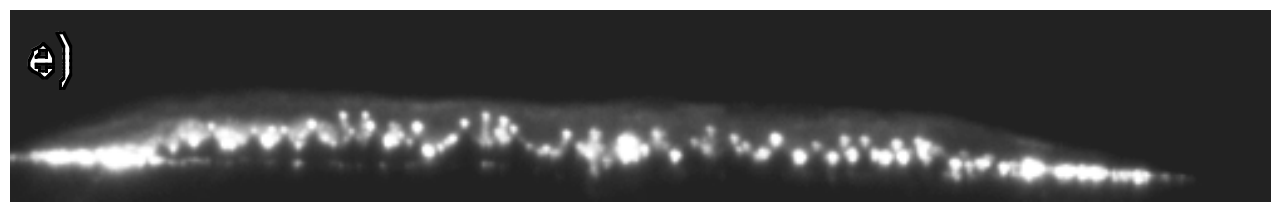

1300 shots

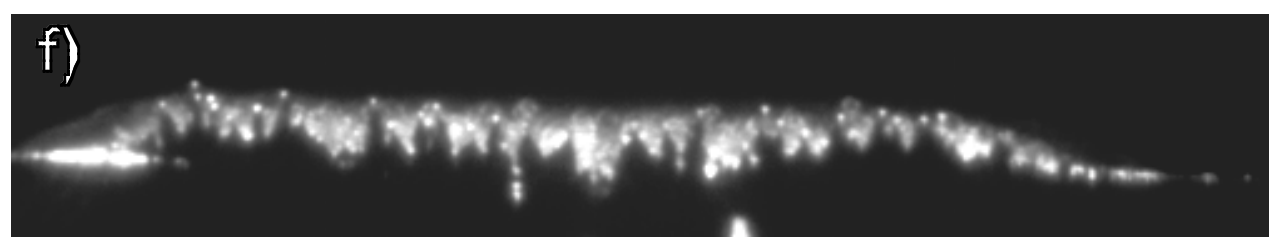

1700 shots

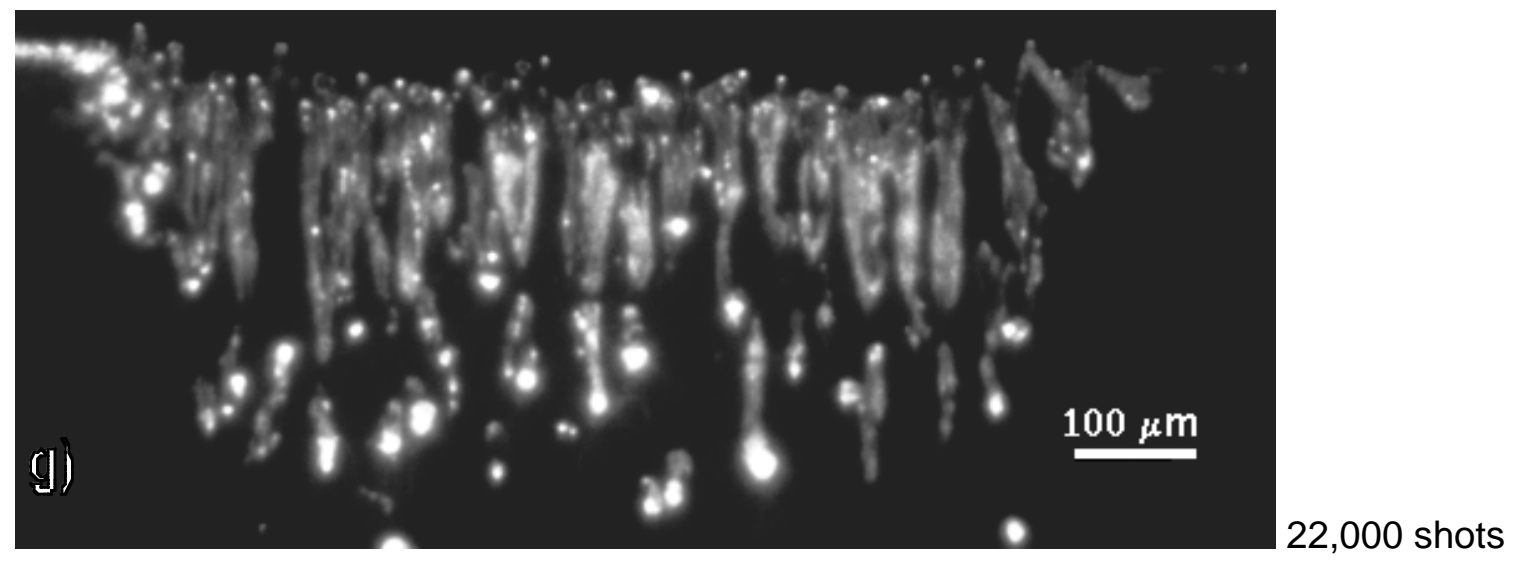

Figure 1. Evolution of microcones and microholes in silicon irradiated at $3.1 \mathrm{~J} / \mathrm{cm}^{2}$ in $\mathrm{SF}_{6}$ at a pressure of 500 mbar. These series of images were taken in-situ with an ICCD camera attached to a long focal distance microscope. The fluorescent plasma seen in the figures is associated to the growth and dissolution of the cones as well as the deepening of the microholes. Figure 1a, 1b, 1c, 1d, 1e, 1f and 1g show the microstructure and plume with a delay time of $55 \mathrm{~ns}$, after 600, 700, 800, 900, 1300, 1700 and 22000 pulses, respectively. 


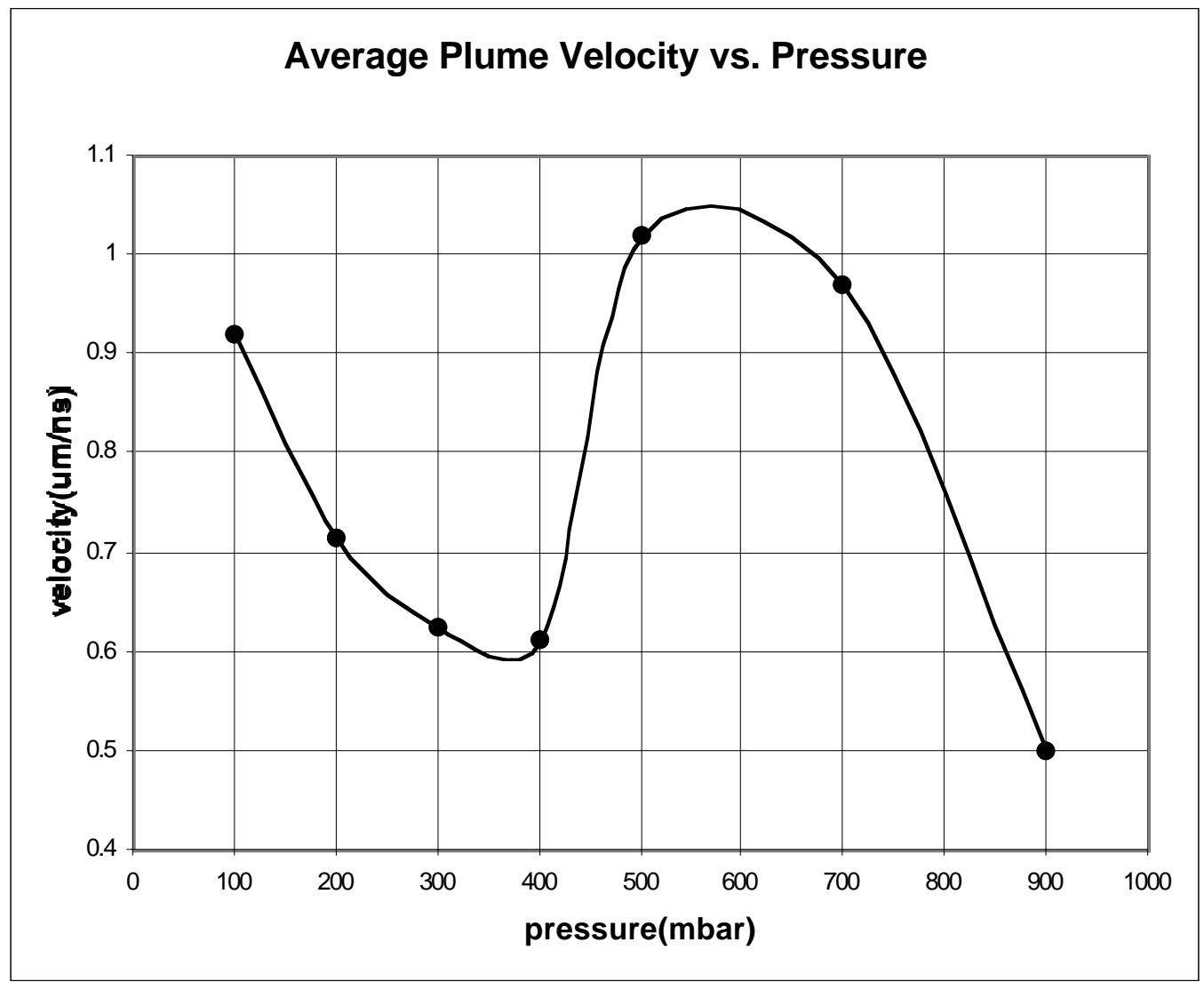

Figure 2. Average plume velocity in the first $100 \mathrm{~ns}$ as a function of $\mathrm{SF}_{6}$ pressure.

cones is clearly visible. The images obtained with the ICCD camera thus show that the fluorescent plume is correlated with the amount of ablated material.

There is also a strong correlation between the plume and the growth of the cones. The cones grow in the region where the plume is intense. The growth of the cones tends to stop when the plume moves away to another region of the irradiated area. At the same time cone growth increases at the region where the plume is moving in. After 20000 laser pulse, when the plume is not longer present, the cones are ablated and retreat below the initial surface level. These results strongly suggest that silicon-rich molecules and clusters transported by the plume are redeposited on the tip and sides of the cones and are responsible for their growth above the initial surface. Simultaneously, because the tips are exposed to the incoming beam, some ablation must also take place. However, this rate is much slow than deposition rate. When the flux of silicon-rich material ceases due to deepening holes, the balance between redeposition and ablation is tilted toward the latter and the cones begin to recede.

We have found that the maximum height reached by the cones is a function of the $\mathrm{SF}_{6}$ pressure. The maximum height is reached when the pressure is 500 mbar [2]. These results correlate with the fact that both the plume front distance and velocity are at a maximum when the $\mathrm{SF}_{6}$ is 500 mbar. The disappearance of a very significant amount of the excited species after the first $100 \mathrm{~ns}$ is probably due to their extremely short mean free path in the very high pressure atmosphere used in these experiments.

The ambient gas $\mathrm{SF}_{6}$ plays two roles: first it acts as an etcher of silicon and second it exerts the background pressure that tends to hold the expansion of the plume. The increase in the ambient pressure from 300 to 500 mbar produces an increase in etching rate thus increasing the pressure produced by the laser-generated plasma. This increase in pressure due to the ablation products is reflected in an increase in the plume velocity. As the background pressure is further increased to 
900 mbar the role of $\mathrm{SF}_{6}$ as an etcher is counterbalanced by the role as a background gas which tends to slow the plume front.

Geohegan found that the plume front reached an initial velocity of $10 \mu \mathrm{m} / \mathrm{ns}$ during the ablation of $\mathrm{YBa}_{2} \mathrm{Cu}_{3} \mathrm{O}_{7-\mathrm{x}}$ in a 200 mtorr of oxygen [12]. In our conditions, the initial velocity of the plume front is one order of magnitude less that that measured by Geohegan. The reason of this difference is most probably related to the difference in ambient pressure that in our experiments was almost 2000 times larger than in the $\mathrm{YBa}_{2} \mathrm{Cu}_{3} \mathrm{O}_{7-\mathrm{x}}$ experiments. Consistent with these differences, Fig. 2 shows that for pressures lower than 300 mbar, the front velocity increases as the background gas pressure decreases.

The detailed study of the formation of this self-organized structure in silicon produced by laser ablation is not only important for the formation of these microstructures but also for the possibility of forming silicon nanostructures. Earlier studies indicate that crevices produced during laser irradiation of graphite targets strongly enhance the process of clustering of carbon atoms [14]. Likewise the formation of this array of microholes and microcones strongly enhances the formation of silicon clusters. If laser irradiation of microstructured silicon surfaces is performed under $\mathrm{Ar}$ atmospheres, nanoparticles are observed outside the irradiated area. The particle size is a function of the distance from the irradiated spot, decreasing with distance from the exposed area. Detailed studies of the formation of nanoparticles produced by laser ablation and enhanced by laser microstructuring is presented elsewhere [15].

\section{ACKNOWLEDGMENTS}

This research was sponsored by the National Science Foundation Grant N DMR-9901238 and by the Oak Ridge National Laboratory, managed by UT-Battelle, LLC, for the U.S. Department of Energy under contract DE-AC05-00OR22725.

\section{REFERENCES}

1. R. Kelly and A. Miotello, "Mechanisms of Pulsed-Laser Sputtering," p. 55-87 in Pulsed Laser Deposition of Thin Films (D. B. Chrisey and G. K. Hubler, eds.) Wiley, New York, 1994.

2. A.J. Pedraza, J. D. Fowlkes, and D. H. Lowndes, Appl. Phys. Lett. 77 (2000) 3018

3. V.V. Voronov, S.I. Dolgaev, S.V. Lavrishchev, A.A. Lyalin, A.V. Simakin and G.A Shafeev, Physics of Vibrations 7 (1999) 131.

4. A. J. Pedraza, J. D. Fowlkes, and D. H. Lowndes, Appl. Phys. A69 (2000) S731

5. F. Sanchez, J. L. Morenza, and V. Trtik, Appl. Phys. Lett. 75 (1999) 3302

6. A.J. Pedraza, J. D. Fowlkes, and D. H. Lowndes, Appl. Phys. Lett. 74 (1999) 2322

7. D. H. Lowndes, J. D. Fowlkes, and A.J. Pedraza, Appl. Sur. Sci. 154, 647 (2000

8. J. D. Fowlkes, A.J. Pedraza, and D. H. Lowndes, Appl. Phys. Lett. 77 (2000) 1629

9. T-H Her, R. J. Finlay, C. Wu, S. Deliwala, and E. Mazur, Appl. Phys. Lett. 73 (1998) 1673.

10. S. De Unamuno and E. Fogarassy, Appl. Sur. Sci., 36, 1 (1989)

11. R. F. Wood and G.E. Jellison, Jr., p. 165 in Semiconductors and Semimetals Vol. 23 (R.F. Wood, C.W. White and R.T. Young, eds.) Academic Press, Orlando, FL, 1984.

12. D. B. Geohegan, Appl. Phys. Lett. 60 (1992) 2732. D.B. Geohegan, in Pulsed Laser Deposition of Thin Films, ed. by D.B. Chrisey and G.K. Hubler (J. Wiley, New York, 1994), ch. 5, p. 115, and references there in.

13. A. O’Keefe, M. M. Ross and A. P. Baronavski, Chem. Phys. Lett., 130, 17 (1986).

14. J.D. Fowlkes, "Growth of Silicon Microcone/Microcolumn Arrays by Nanosecond Pulsed-Laser Irradiation" M. Sc. Thesis, The University of Tennessee, Knoxville, August 1999.

15. J. D. Fowlkes, A. J. Pedraza and S. Jesse C.M. Rouleau, and D.A. Blom, MRS Vol. 638 (2000) 